\title{
PREVALENCIA DE HIPERTROFIA VENTRICULAR IZQUIERDA EN PACIENTES DIABÉTICOS
}

\author{
Diego Valarezo-Sevilla ${ }^{1, a}$, Armín Pazmiño-Martínez²,a, Nidya Morales-Mora,a
}

\begin{abstract}
RESUMEN
Con el objetivo de establecer la prevalencia de hipertrofia ventricular izquierda $(\mathrm{HVI})$ en pacientes con diabetes mellitus tipo 2 (DM), se realizó un estudio transversal en estos pacientes, estableciendo sus características antropométricas, presión arterial y control metabólico. Para evaluar la presencia de HVI se empleó ecocardiografía transtorácica. El estudio incluyó 91 pacientes, en los cuales la prevalencia de HVI fue de $63,7 \%$, siendo más frecuente en mujeres que en varones $(p=0,001)$. Adicionalmente, se encontró un $46,2 \%$ de pacientes con disfunción diastólica del ventrículo izquierdo. Se concluye que existe una importante prevalencia de $\mathrm{HVI}$ en pacientes diabéticos sin antecedentes de causas definidas de hipertrofia. No se encontró relación con sexo, control metabólico, IMC y tiempo de diagnóstico.
\end{abstract}

Palabras clave: Diabetes mellitus; Hipertrofia ventricular izquierda; Ecocardiografía (fuente: DeCS BIREME).

\section{PREVALENCE OF LEFT VENTRICULAR HYPERTROPHY IN DIABETIC PATIENTS}

\begin{abstract}
In order to establish the prevalence of left ventricular hypertrophy (LVH) in patients with type 2 diabetes mellitus, (DM) a cross-sectional study was conducted in these patients studying their anthropometric characteristics, blood pressure and metabolic control. To evaluate the presence of $\mathrm{LVH}$, a trans-thoracic echocardiogram was used. The study included 91 patients, finding a $63.7 \%$ prevalence of $\mathrm{HVI}$, with women being more affected than men $(p=0.001)$. Additionally, $46.2 \%$ of patients were found to have diastolic dysfunction of the left ventricle. We conclude that there is an important prevalence of LVH in diabetic patients without defined causes of hypertrophy. There was no association with sex, metabolic control, BMl and time of diagnosis.
\end{abstract}

Key words: Diabetes Mellitus; Hypertrophy, left ventricular; Echocardiography (source: MeSH NLM).

\section{INTRODUCCIÓN}

La hipertrofia de ventrículo izquierdo (HVI) es un factor de riesgo cardiovascular independiente, la cual está vinculada a varios mecanismos fisiopatológicos subyacentes como el deterioro de la función sistólica y diastólica, y la disminución de la reserva coronaria en ausencia de estenosis coronaria. Evidentemente la reducción de HVI reduce también el riesgo cardiovascular $(1,2)$. La hipertensión arterial (HTA) es la principal entidad clínica capaz de causar daño en la función ventricular, más aun si se presenta simultáneamente con otros males como las nefropatías o las enfermedades metabólicas ${ }^{(3,4)}$. Por otro lado, la diabetes mellitus tipo 2 (DM) es una de las enfermedades metabólicas con más alta prevalencia a nivel mundial, ella también está asociada a un mayor riesgo cardiovascular mediado básicamente por el daño que se produce en el endotelio vascular. Aunque pocos, existen estudios que señalan la relación entre la DM, como factor independiente, y la aparición de $\mathrm{HVI}$. Eguchi et al. concluyeron que la presencia de DM incrementa el riesgo de presentar HVI en 1,5 veces ${ }^{(5)}$,

\footnotetext{
Hospital Básico Jipijapa. Jipijapa, Ecuador.

2 Clínica San Francisco. Sangolquí, Ecuador

3 Hospital de Latacunga. Latacunga, Ecuador.

a Médico internista

Los resultados provienen de la tesis para optar por el grado de especialista en Medicina Interna realizada en el Instituto Superior de Postgrado de la Facultad de Ciencias Médicas de la Universidad Central del Ecuador; 2010.

Recibido: 01-11-12 Aprobado: 20-02-13
}

Citar como: Valarezo-Sevilla D, Pazmiño-Martínez A, Morales-Mora N. Prevalencia de hipertrofia ventricular izquierda en pacientes diabéticos. Rev Peru Med Exp Salud Publica. 2013;30(1):69-72. 
en dicho estudio, además, se señala que la obesidad central cumple también un rol en la aparición de la HVI. Por otro lado, el Strong Heart Study encontró que las mediciones ecocardiográficas de la masa del ventrículo izquierdo catalogadas para el área de superficie corporal o peso fueron superiores 9 a $14 \%$, y 6 al $12 \%$, respectivamente en mujeres y hombres diabéticos comparados con tolerantes a la glucosa siendo esta diferencia más importante en mujeres ${ }^{(6)}$.

Dada la importancia de la prevención y el control del riego cardiovascular en las enfermedades crónicas no trasmisibles, es que se plantea el presente trabajo de investigación cuyo objetivo fue determinar la prevalencia de hipertrofia ventricular izquierda en pacientes con DM.

\section{EL ESTUDIO}

Se realizó un estudio transversal en el Hospital General de las Fuerzas Armadas N. 1 (HG-1) de QuitoEcuador. La población objetivo estuvo conformada por pacientes con DM de la consulta externa del servicio de endocrinología. Se consideró como criterios de inclusión a todo paciente atendido entre enero de 2004 y diciembre de 2009, que tuviese el diagnóstico de DM. El Departamento de Estadística del HG-1 reportó para este periodo un total de 563 pacientes, se excluyeron 384 pacientes con antecedentes de causas conocidas de HVI como enfermedad valvular aórtica, insuficiencia renal, arritmia cardíaca, mayores de 75 años, HTA previamente diagnosticada, y pacientes en uso de antihipertensivos, por lo que la dimensión de nuestro marco muestral fue de 179 pacientes, para el cálculo del tamaño muestral se consideró una prevalencia de $9 \%$ (prevalencia encontrada en el Strong Heart Study ${ }^{(6)}$ ), con un nivel de confianza del 95\% y una precisión del $5 \%$, con lo cual se estableció un tamaño muestral final de 75 pacientes. La selección de pacientes se realizó con una tabla de números aleatorios, durante los meses de mayo a agosto de 2010, en la consulta externa del servicio de Endocrinología.

Para el diagnóstico de DM se tomaron en cuenta los criterios de laAmerican Diabetes Association del 2009 (7), el tiempo de diagnóstico se registró en años. Se realizaron ecocardiografías en modo bidimensional (sensibilidad del $85 \%$, especificidad del $97 \%$, valor predictivo positivo del $98 \%$ y valor predictivo negativo del $94 \%$ ) ${ }^{(8,9)}$, las cuales fueron realizadas por un cardiólogo con formación especializada en ecocardiografía. Para medir la masa ventricular se empleó las fórmulas validadas por la Sociedad Americana de Ecocardiografía, el diagnóstico de HVI se realizó en base al índice de masa ventricular izquierdo (IMVi), el cual se calcula dividiendo la masa ventricular izquierda entre la superficie corporal total; se consideró $\mathrm{HVI}$ en quienes presentaban un IMVi mayor a $95 \mathrm{~g} / \mathrm{m}^{2}$ en mujeres y mayor a $115 \mathrm{~g} / \mathrm{m}^{2}$ en varones ${ }^{(10)}$. De manera adicional se evaluó la función diastólica, se determinó su disfunción al encontrar alteraciones en el patrón de flujo transmitral tales como patrón de relajación lenta, patrón de llenado pseudonormal o patrón de llenado restrictivo ${ }^{(11)}$. Se realizaron, además, mediciones de peso, talla, índice de masa corporal (IMC), perímetro abdominal, control de presión arterial y electrocardiograma (para descartar arritmias). De las historias clínicas se obtuvieron los valores del último control de hemoglobina glucosilada (HbA1c).

La variable edad fue dicotomizada en las categorías "adulto" y "adulto mayor" tomando como punto de corte los 65 años. Para la descripción de las variables categóricas se calcularon las frecuencias relativas y absolutas, con sus respectivos intervalos de confianza. Para evaluar la asociación entre las variables se empleó la prueba chi cuadrado, se fijó el nivel de confianza al $95 \%$ y el error alfa en $5 \%$. El presente estudio contó con la autorización del comité de Bioética del HG-1 (N. ${ }^{\circ}$ 10-017HG-1-10CBE), así como el consentimiento informado firmado por cada uno de los pacientes que intervinieron.

\section{HALLAZGOS}

Se evaluó un total de 91 pacientes, la media de edad fue $59,3 \pm 11,3$ años; el $52,7 \%$ fue de sexo femenino. La media del tiempo de enfermedad fue de 3,6 $\pm 2,1$ años. La media del IMC fue de $29,4 \pm 4,7 \mathrm{~kg} / \mathrm{m}^{2}$, siendo mayor en mujeres $\left(30,3 \pm 5,2 \mathrm{~kg} / \mathrm{m}^{2}\right)$ que en varones $(28,3 \pm$ $3,9 \mathrm{~kg} / \mathrm{m}^{2}$ ). La media de la presión arterial sistólica (PAS) fue de $118,2 \pm 10,3 \mathrm{mmHg}$ y de la presión arterial diastólica (PAD) fue de 75,7 $\pm 7,6 \mathrm{mmHg}$. La frecuencia de HVI para la población estudiada fue de $63,7 \%$ [IC: 52,3-72,9], demostrándose ser más frecuente en mujeres $65,5 \%$ en mujeres y $34,5 \%$ en varones $(p=0,001)$.

En nuestra muestra se encontró, además, relación entre la HVI y la edad $(p=0,002)$, con mayor prevalencia en adultos mayores $(76,5 \%)$ que en el resto de la población estudiada. No se encontró relación entre el tiempo de enfermedad y la presencia de HVI $(p=0,6)$. En el grupo de pacientes con $\mathrm{HVI}$ se observó un mayor promedio de $\mathrm{HbA} 1 \mathrm{c}$ de $7,6 \pm 2,1 \%$, siendo mayor en varones $7,8 \pm$ $1,8 \%$, que en mujeres $7,1 \pm 0,9 \%$; no se halló relación entre la presencia de HVI y la variable HbA1c. Durante el estudio se identificaron a 42 personas con disfunción diastólica de ventrículo izquierdo $(46,1 \%)$, de los cuales el $57,1 \%$ eran varones, sin encontrarse asociación con el sexo $(p=0,06)$. 


\section{DISCUSIÓN}

En la literatura biomédica existen escasos estudios sobre $\mathrm{HVI}$ en pacientes diabéticos en ausencia de HTA ${ }^{\left({ }^{12}\right)}$. En el trabajo de Dawson et al. ${ }^{(3)}$ el $61 \%$ de diabéticos tuvieron HTA y en el estudio de Framingham ${ }^{(13)}$ se encontró HVI en el $28 \%$ de mujeres sobre los 60 años con presiones arteriales sistólicas mayores de $125-139 \mathrm{mmHg}$. En otro estudio publicado recientemente se encontró que el $60 \%$ de diabéticos estudiados tenía HTA y el $56 \%$ $\mathrm{HVI}$, de las cuales solo el $37 \%$ de los pacientes no tenía antecedentes de HTA ${ }^{(14)}$.

Por ello cobran especial interés las investigaciones que tienen como objetivo estudiar la relación existente entre la HVI y la DM en ausencia de otros factores cardiovasculares; en este trabajo se encontró una frecuencia de $63,7 \%$ de $\mathrm{HVI}$ en pacientes diabéticos sin antecedentes de causas definidas para esta enfermedad; valor que coincide con otros estudios que informan porcentajes similares en diferentes poblaciones (12,13,15); lo cual refuerza la hipótesis planteada. Esta investigación sugiere, además, que los adultos mayores del sexo femenino que padecen de DM son el grupo de riesgo más vulnerable a desarrollar $\mathrm{HVI}$, tal como lo sugieren otros estudios (16,17). De manera adicional, es necesario decir que la frecuencia que se ha encontrada en la muestra trabajada, es mayor a la informada por estudios en poblaciones americanas y españolas ${ }^{(15,17)}$. En el primero de estos estudios se buscó $\mathrm{HVI}$ en pacientes diabéticos, y en el segundo en pacientes con HTA, en este último se realizó un análisis estratificado según el diagnóstico de DM, encontrando una mayor prevalencia de HVI en los pacientes diabéticos de su muestra. En relación a este punto se debe añadir que la mayor frecuencia encontrada en este estudio se podría deber a que los pacientes diabéticos incluidos tuvieron características particulares que los predispusieron a desarrollar más rápidamente esta enfermedad, entre las cuales podemos incluir factores genéticos, dietéticos, adherencia al tratamiento, tiempo de diagnóstico de la enfermedad o mejor control metabólico.

Otro factor a tomar en cuenta es la obesidad, la cual también es considerada como un factor independiente de aumento de tamaño de cámara cardiaca, del grosor de la pared de ventrículo izquierdo y de la masa ventricular izquierda. Un estudio reciente encontró $\mathrm{HVI}$ en $23 \%$ de los pacientes diabéticos estudiados, independientemente de HTA concomitante, de estos pacientes la mayoría fueron mujeres y presentaban además un mayor IMC (17), con lo cual se puede suponer que la obesidad podría modificar la relación existente entre HVI y DM. En el Strong Heart Study la población tuvo un IMC promedio de $32,4 \pm 6,5 \mathrm{~kg} / \mathrm{m} 2$, es decir, obesidad grado I, mientras que Dawson et al. encontraron un promedio de IMC de $29,8 \pm 5,1 \mathrm{~kg} / \mathrm{m}^{2}$, es decir, se encontraban en sobrepeso. En este estudio la media del IMC fue de $29,4 \pm 4,7 \mathrm{~kg} / \mathrm{m}^{2}$, con lo que podemos afirmar que la obesidad no constituye, en esta muestra, un factor modificador en la relación existente entre la HVI y la DM, siendo que las frecuencias encontradas aquí son mayores, a pesar de que esta muestra tiene, en promedio, un menor peso.

En relación al control metabólico, no existió relación entre la presencia de $\mathrm{HVI}$ y valores de $\mathrm{HbA} 1 \mathrm{c}$, hecho que coincide con Dawson et al. donde tampoco se encontró asociación con esta variable ${ }^{(18)}$. La concordancia de estos hallazgos nos hace pensar que probablemente la HbA1c sea un factor muy importante en relación a las complicaciones cardiovasculares de la DM, no así en el caso de HVI. Es necesario recalcar que no se encontró asociación entre HVI y el tiempo de evolución de diabetes, que es otro factor identificado en la aparición de complicaciones crónicas cardiovasculares y no cardiovasculares.

Finalmente, en el presente trabajo, durante la evaluación ecocardiográfica, se encontró una importante frecuencia de disfunción diastólica de ventrículo izquierdo en diabéticos, sin existir asociación con el sexo, con el tiempo de diagnóstico, ni con el control metabólico, si bien es cierto este valor concuerda con el $41 \%$ hallado por Dawson et al. se requiere mayores estudios que busquen investigar esta relación.

A pesar de la alta prevalencia encontrada de hipertrofia ventricular en los pacientes diabéticos en este y otros estudios, desafortunadamente no es posible aun recomendar el uso rutinario de la ecocardiografía en estos pacientes debido a la baja accesibilidad a este examen, sobre todo en niveles de atención primaria. Consideramos que se debe seguir investigando sobre el tema, realizando estudios costo-beneficio en pacientes diabéticos para determinar, entre las opciones diagnósticas ${ }^{(18)}$, la más adecuada para poder realizar un tamizaje de la HVI.

No se puede dejar de mencionar que en este trabajo las principales limitaciones son el tamaño de la muestra y su nivel de inferencia, aun cuando se trata de un muestreo probabilístico el nivel de inferencia es solo el hospital en el cual se realizó el estudio. Ahora bien, siendo el HG-1 un hospital de alta complejidad y centro nacional de referencias es evidente que existen un número de pacientes con DM que no han sido incluidos en la muestra puesto que se deben de atender en establecimientos de menor capacidad resolutiva. 
En conclusión, se encontró una alta frecuencia de HVI en pacientes diabéticos, en ausencia de otros factores cardiovasculares que pudieron haberla causado, en donde, la población adulto mayor de sexo femenino es la más vulnerable a desarrollar esta enfermedad. Aun cuando la ecografía es el método de diagnóstico más adecuado para realizar el diagnostico de HVI, se considera que hacen falta estudios costo-beneficio para poder recomendar una prueba de tamizaje para HVI en pacientes diabéticos.

Agradecimientos: a los doctores Rodrigo Rovayo y Washington Paz por su colaboración en la dirección científica y metodológica del estudio, así como al Dr. Hugo Aucancela quien labora en el Hospital General de las Fuerzas Armadas N. ${ }^{\circ} 1$ de Quito por su ayuda en la realización de las ecocardiografías.

Contribuciones de autoría: DVS, APM, NMM participaron en la concepción y diseño del artículo, en la recolección y obtención de resultados, análisis e interpretación de datos, así como en la redacción del artículo y revisión crítica del artículo. Todos los autores participaron de la aprobación de la versión final del artículo.

Fuentes de financiamiento: autofinanciado.

Conflictos de interés: los autores declaran no tener conflictos de interés.

\section{REFERENCIAS BIBLIOGRÁFICAS}

1. Lorell B, Carabello B. Left ventricular hypertrophy: pathogenesis, detection, and prognosis. Circulation. 2000;102(4):470-9

2. Sato A, Tarnow L, Parving $\mathrm{HH}$. Prevalence of left ventricular hypertrophy in type I diabetic patients with diabetic nephropathy. Diabetologia. 1999;42(1):76-80.

3. Dawson A, Morris AD, Struthers AD. The epidemiology of left ventricular hypertrophy in type 2 diabetes mellitus. Diabetologia. 2005;48(10):1971-9

4. Codinach P, Freixa R. Miocardiopatía diabética: concepto, función cardiaca y patogenia. An Med Interna. 2002;19(6):313-20.

5. Eguchi K, Boden-Albala B, Jin Z, Rundek T, Sacco R, Homma S, et.al. Association between diabetes mellitus and left ventricular hypertrophy in a multi-ethnic population. Am J Cardiol. 2008;101(12):1787-91.

6. Bella JN, Devereux RB, Roman MJ, Palmieri V, Liu JE, Paranicas M, et al. Separate and joint effects of systemic hypertension and diabetes mellitus on left ventricular structure and function in American Indians (the Strong Heart Study). Am J Cardiol. 2001;87(11):1260-5.

7. American Diabetes Association. Standards of medical care in diabetes--2009. Diabetes Care. 2009;32 Suppl 1:S13-61.

8. Devereux RB, Alonso DR, Lutas EM, Gottlieb GJ, Campo E, Sachs I, et al. Echocardiographic assessment of left ventricular hypertrophy: comparison to necropsy findings. Am J Cardiol. $1986 ; 57(6): 450-8$

9. Erbel R, Schweizer P, Krebs W, Meyer J, Effert S. Sensitivity and specificity of two-dimensional echocardiography in detection of impaired left ventricular function. Eur Heart J. 1984;5(6):477-89.

10. Lang RM, Bierig M, Devereux RB, Flachskampf FA, Foster E, Pellikka $\mathrm{PA}$, et al. Recommendations for chamber quantification: a report from the American Society of Echocardiography's Guidelines and Standards Committee and the Chamber Quantification Writing Group, developed in conjunction with the European Association of Echocardiography, a branch of the European Society of Cardiology. J Am Soc Echocardiogr. 2005;18(12):1440-63.

11. Carrillo R, Contreras NA. Conceptos actuales en disfunción diastólica. Rev Asoc Mex Med Crit y Ter Int. 2008;22(3):163-73.

12. Santra S, Basu AK, Roychowdhury P, Banerjee R, Singhania P, Singh S, et al. Comparison of left ventricular mass in normotensive type 2 diabetes mellitus patients with that in the nondiabetic population. J Cardiovasc Dis Res. 2011;2(1):50-6.

13. Rutter M, Parise H, Benjamin E, Levy $\mathrm{D}$, Larson $\mathrm{M}$, Meigs J, et al. Impact of glucose intolerance and insulin resistance on cardiac structure and function: sex-related differences in the Framingham Heart Study. Circulation. 2003;107(3):448-54.
14. Somaratne JB, Whalley GA, Bagg W, Doughty RN. Early detection and significance of structural cardiovascular abnormalities in patients with Type 2 diabetes mellitus. Expert Rev Cardiovasc Ther. 2008;6(1):109-25.

15. Devereux R, Roman M, Paranicas M, O'Grady M, Lee E, Welty T, et al. Impact of diabetes on cardiac structure and function: the strong heart study. Circulation. 2000;101(19):2271-6.

16. Cioffi G, Faggiano P, Lucci D, Di Lenarda A, Mureddu GF, Tarantini $\mathrm{L}$, et al. Inappropriately high left ventricular mass in patients with type 2 diabetes mellitus and no overt cardiac disease. The DYDA study. J Hypertens. 2011;29(10):1994-2003.

17. Lozano J, Redón J, Cea-Calvo L, Fernández-Pérez C, Navarro J, Bonet A, et al. Hipertrofia ventricular izquierda en la población hipertensa española. Estudio ERIC-HTA. Rev Esp Cardiol. 2006;59(2):136-42.

18. Somaratne JB, Whalley GA, Poppe KK, ter Bals MM, Wadams G, Pearl A, et al. Screening for left ventricular hypertrophy in patients with type 2 diabetes mellitus in the community. Cardiovasc Diabetol. 2011;10:29

Correspondencia: Diego Valarezo Sevilla Dirección: Sánchez y Cifuentes 20-64 y Ricardo Sánchez. Ibarra, Ecuador. Teléfono: (593) 998393747

Correo electrónico: valarezodiego_md@hotmail.com 